\title{
Optical Control of Cardiac Function with a Photoswitchable Muscarinic Agonist
}

\author{
Fabio Riefolo ${ }^{1,2, \dagger}$, Carlo Matera ${ }^{1,2,}$, , Aida Garrido-Charles ${ }^{1,2}$, Alexandre M. J. Gomila ${ }^{1,2}$, Rosalba Sortino ${ }^{1,2}$, Luca Agnetta ${ }^{3}$, \\ Enrique Claro ${ }^{4}$, Roser Masgrau ${ }^{4}$, Ulrike Holzgrabe ${ }^{3}$, Montserrat Batlle ${ }^{5}$, Michael Decker ${ }^{3}$, Eduard Guasch ${ }^{5,6}$, and Pau Goros- \\ tiza $1,2,7, *$
}

\begin{abstract}
Light-triggered reversible modulation of physiological functions offers the promise of enabling on-demand spatiotemporally controlled therapeutic interventions. Optogenetics has been successfully implemented in the heart, but significant barriers to its use in the clinic remain, such as the need for genetic transfection. Herein, we present a method to modulate cardiac function with light through a photoswitchable compound and without genetic manipulation. The molecule, named PAI, was designed by introduction of a photoswitch into the molecular structure of an M2 mAChR agonist. In vitro assays revealed that PAI enables light-dependent activation of M2 mAChRs. To validate the method, we show that PAI photoisomers display different cardiac effects in a mammalian animal model, and demonstrate reversible, real-time photocontrol of cardiac function in translucent wildtype tadpoles. PAI can also effectively activate M2 receptors using two-photon excitation with near-infrared light, which overcomes the scattering and low penetration of short-wavelength illumination, and offers new opportunities for intravital imaging and control of cardiac function.
\end{abstract}

cardiac photoregulation, cholinergic, muscarinic, photopharmacology, dualsteric agonist, two-photon

\section{Introduction}

Remote spatiotemporal control of physiological processes may provide novel treatment opportunities. Cardiopathies are paradigmatic in this regard because of the rapid time course and complex integration of electrophysiological and molecular events in very specific areas of the heart. For instance, most cardiac rhythm control strategies rely on antiarrhythmic drugs (AADs) targeting ionic currents, whose effects cannot be regulated spatiotemporally. As a result, AADs often give rise to intolerable side effects, including ventricular pro-arrhythmogenicity, and are only partially effective. Overcoming the high failure and complication rates of current therapeutic strategies to treat these diseases will require both patient-personalized determination of the specific physiopathological mechanism and qualitative pharmacological breakthroughs. ${ }^{1}$

The application of light and optical techniques in medicine has had a profound impact over the last several decades, in diagnostics, surgery and therapy. ${ }^{2}$ In particular, photoexcitation of intrinsic molecules or exogenous light-sensitive agents introduced in the body can affect the tissues and cells within in various ways, via the generation of heat (photothermal), chemical reactions (photochemical), and biological processes (photobiological/photopharmacological or optogenetic). ${ }^{2}$ The potential of light as a

Institute for Bioengineering of Catalonia (IBEC), Barcelona Institute for Science and Technology (BIST).

2. Network Biomedical Research Center in Bioengineering, Biomaterials, and Nanomedicine (CIBER-BBN).

Pharmaceutical and Medicinal chemistry, Institute of Pharmacy and Food Chemistry, Julius Maximilian University of Würzburg, Am Hubland, D-97074 Würzburg, Germany.

4. Institut de Neurociències (INc), and, Dept. Bioquímica i Biologia Molecular, Unitat de Bioquímica de Medicina, Universitat Autònoma de Barcelona (UAB).

Cardiovascular Institute, Hospital Clinic; University of Barcelona (UB); IDIBAPS.

6. Network Biomedical Research Center in Cardiovascular Diseases (CIBER$\mathrm{CV}$ ).

Catalan Institution for Research and Advanced Studies (ICREA).

$\dagger$ Contributed equally to this work.

* Corresponding author. therapeutic tool with high spatiotemporal resolution has been recently investigated in the cardiovascular field, particularly for arrhythmias, through optogenetics. ${ }^{3-7}$ However, the application of such genetic techniques to human subjects with therapeutic purposes is still hampered by safety, regulatory and economic hurdles. Unlike optogenetics, photopharmacology rely on the use of exogenous light-regulated small molecules that can photocontrol native targets and that could be tested and approved using standard drug development procedures. ${ }^{8-15}$ These molecules can be used in combination with devices that deliver light to specific locations in the body ${ }^{7,16-18}$ in order to remotely control drug dosing and duration of action. Since the activity of drugs is structure-dependent, reversible photoresponsive drugs are obtained by the rational introduction of a molecular photoswitch into the structure of a bioactive compound. .,14 $^{8}$

Cardiac function is controlled by the autonomic sympathetic and parasympathetic nervous systems, which act via adrenoceptors and muscarinic acetylcholine receptors (mAChRs), respectively. ${ }^{19}$ In particular, stimulation of $\beta 1$ and $\beta 2$ adrenergic receptors increases the heart rate (positive chronotropy) and contractility (positive inotropy), whilst stimulation of M2 mAChRs decreases heart rate and prolongs the atrioventricular conduction time. ${ }^{20,21}$ Thus, adrenergic and muscarinic receptors constitute suitable target candidates to control cardiac function with light. Muscarinic acetylcholine receptors ( $\mathrm{mAChRs}$ ) belong to class A G protein-coupled receptors (GPCRs) and are divided in five different subtypes (M1-M5). ${ }^{22} \mathrm{M} 2$ receptor is extensively expressed in the heart. All five $\mathrm{mAChRs}$ are characterized by a high sequence homology in the orthosteric site located in the transmembrane region. This fact limits the development of subtype-selective orthosteric agonists. On the other hand, the allosteric site located in the extracellular loop is less conserved, thus muscarinic allosteric agents are commonly endowed with a more pronounced subtype-selectivity. ${ }^{23} \mathrm{~A}$ chemical strategy commonly applied to overcome such limitation is the incorporation, within the same molecular structure, of two distinct pharmacophore elements belonging to (a) high-affinity orthosteric agonists and (b) highly selective allosteric ligands. ${ }^{24-28}$ 


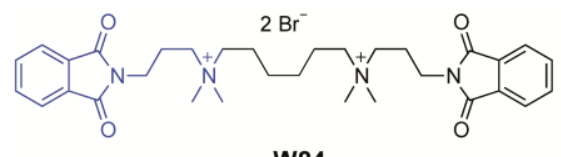

W84

(allosteric M2 mAChR modulator)

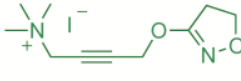

Iperoxo

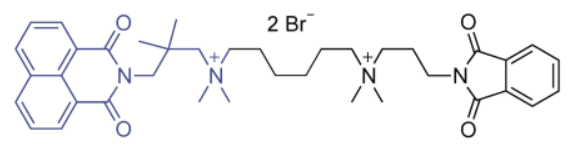

Naphmethonium

(allosteric M2 mAChR modulator)

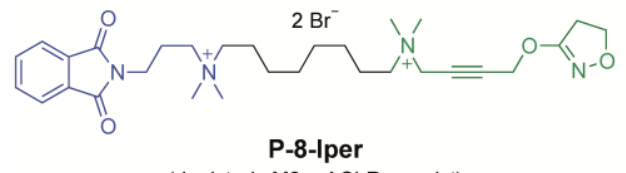

(dualsteric M2 mAChR agonist)

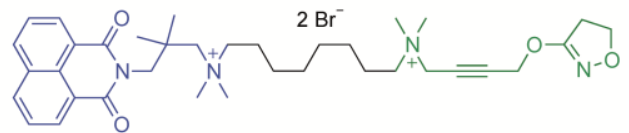

N-8-Iper

(dualsteric M2 mAChR agonist)

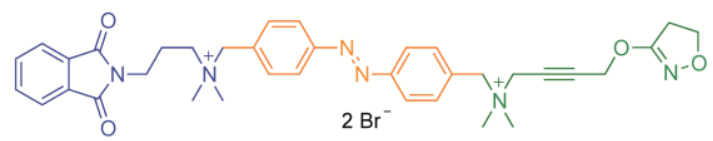

PAI (1)

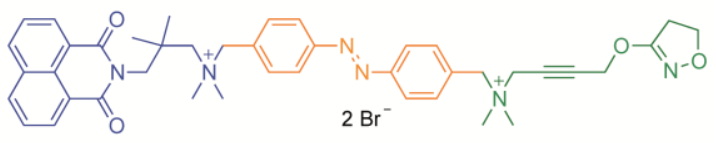

NAI (2)

Fig. 1. Chemical structures of the muscarinic ligands discussed in this work: the allosteric modulators W84 and Naphmethonium, the orthosteric agonist Iperoxo, the dualsteric agonists P-8-Iper and N-8-Iper, and the photoswitchable dualsteric ligands PAI and NAI.

These hybrid compounds, termed "dualsteric" or "bitopic", are capable to bind simultaneously to both the orthosteric and the allosteric sites of mAChRs and usually display valuable properties, ${ }^{29}$ such as receptor subtype selectivity, functional selectivity, ${ }^{26,30}$ and higher tolerability in vivo. ${ }^{31}$ Moreover, such ligands bear in their structure two permanently charged nitrogen atoms, which likely prevent them from crossing the blood-brain barrier, confining their effects to the periphery, which is advantageous for cardiovascular agents. Herein, we describe the first non-genetic method for the optical control of cardiac function with a photoswitchable agonist. The design and synthesis of this molecule and its pharmacological characterization under one-photon (1P) and twophoton (2P) excitation are reported.

\section{Results and Discussion}

Rational design, chemical synthesis and photochemical characterization. Two novel putative photoresponsive muscarinic agents, named Phthalimide-Azo-Iperoxo (PAI) and NaphthalimideAzo-Iperoxo (NAl), were designed by replacing the polymethylene spacer chain of known M2 dualsteric agonists (P-8-Iper and N-8Iper) ${ }^{30-32}$ with a molecular photoswitch, while conserving (a) the
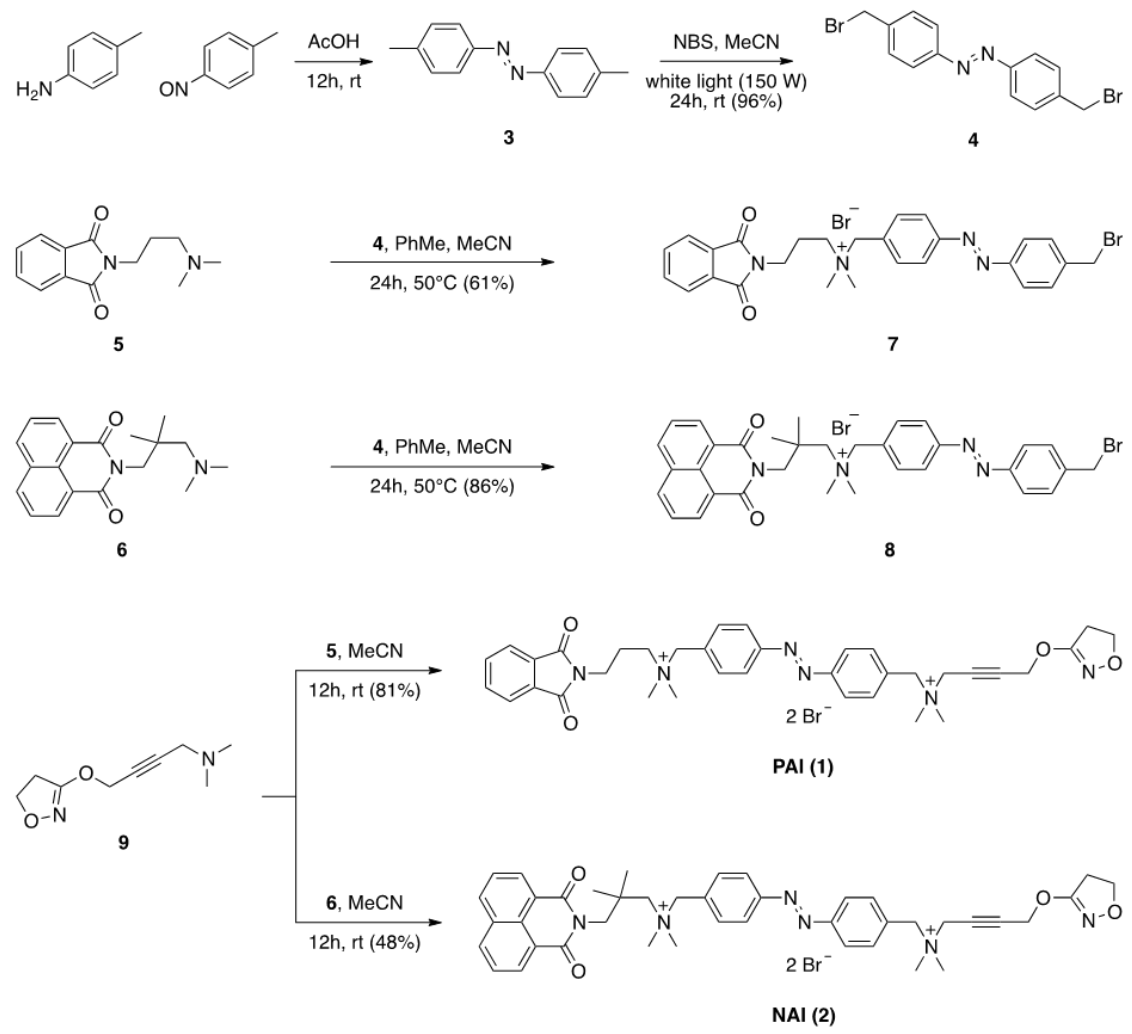

Scheme 1. Chemical synthesis of PAI and NAI. 
a

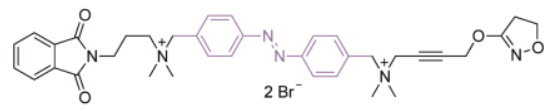

trans-PAI

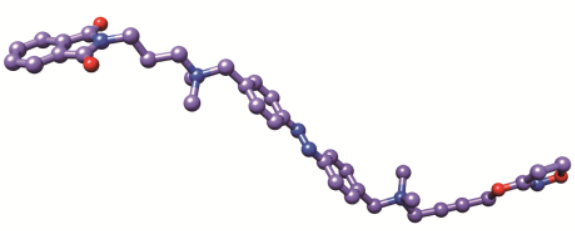

b

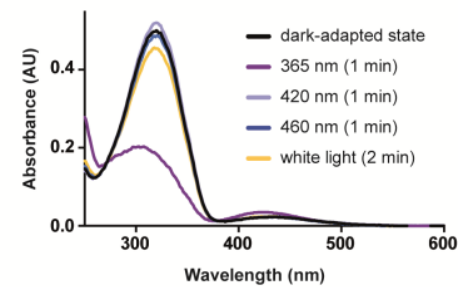

C

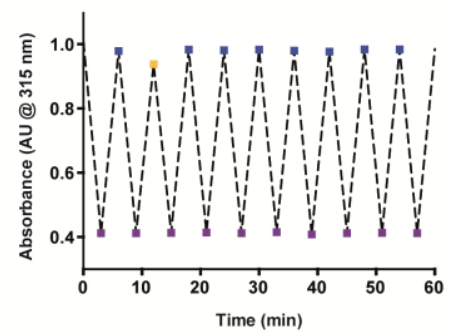

Fig. 2. Photochemical characterization. a) $2 \mathrm{D}$ and $3 \mathrm{D}$ representation of the chemical structures of trans- and cis-PAI. b) Absorption spectra of PAI showing distinct photochromic behaviour. c) The photoswitching process can be repeated over several cycles without noticeable photofatigue.

Iperoxo-like orthosteric agonist moiety, and (b) the M2-selective allosteric fragments derived from W84 and Naphmethonium (Fig. 1). The incorporation of a photoisomerizable unit into the structure of a dualsteric agonist should enable controlling with light the mutual position of the orthosteric and the allosteric moieties, presumably leading to differences between the two isomers in receptor affinity and efficacy. We chose an azobenzene core as photoresponsive component because of the favourable characteristics
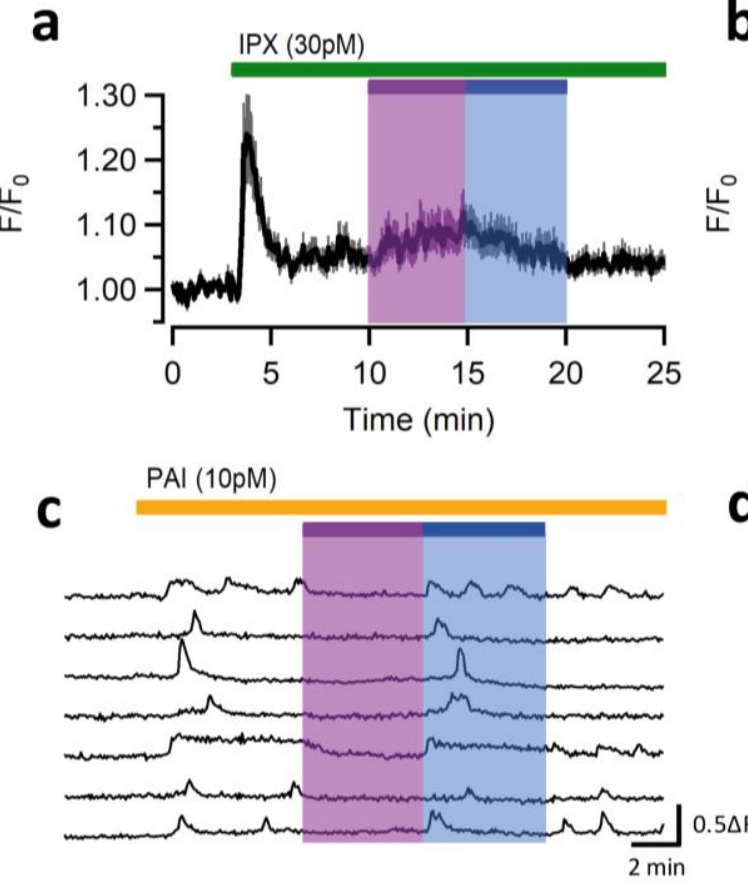

that azobenzene-based photoswitches normally display for biological purposes in comparison to other photoswitches, such as design flexibility, large changes in geometry upon isomerization, high photostationary states and fatigue resistance, fast photoisomerization rates, and chemical stability, among others. ${ }^{33}$ Moreover, the use of arylazo compounds has been proven safe in humans for some approved drugs and food colorants. ${ }^{33}$

b

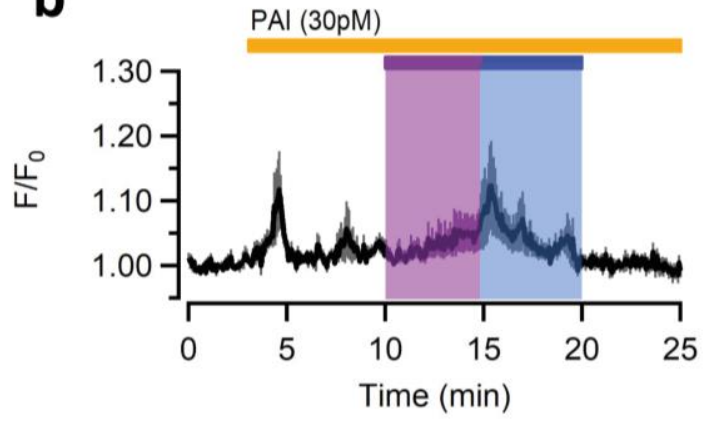

d

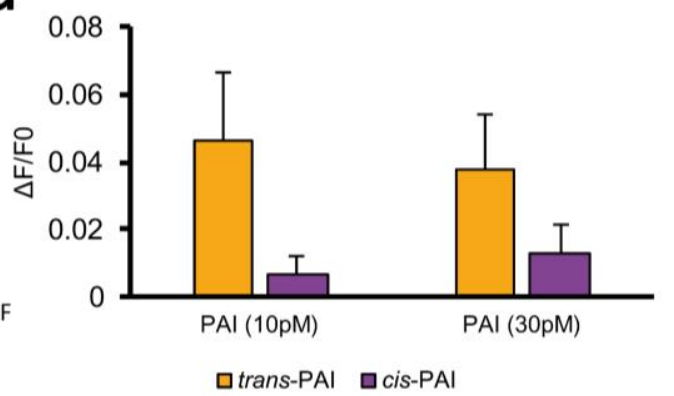

Fig. 3. PAI reversibly activates $\mathrm{M} 2 \mathrm{mAChRs}$ with light in calcium imaging assays. Real-time calcium imaging traces from HEK cells co-expressing $\mathrm{M} 2 \mathrm{mAChR}$ and GqTOP loaded with $10 \mu \mathrm{M}$ of the calcium indicator OGB1AM. a) Average trace of cell responses to $30 \mathrm{pM}$ of non-photoswitchable muscarinic agonist IPX $(\mathrm{n}=130)$. Cells gave a sharp response to the application of IPX but, as expected, the concomitant application of pulses of UV or blue light did not alter calcium responses. b) Average trace of cell responses to $30 \mathrm{pM}$ of trans-PAI $(\mathrm{n}=8)$. c) Single cell calcium responses induced by the direct application of $10 \mathrm{pM}$ of trans-PAI (yellow bar). Purple bars indicate illumination at $365 \mathrm{~nm}$, blue bars indicate illumination at $460 \mathrm{~nm}$. Gray shadow in the recordings represents \pm SEM. d) Quantification of photoresponses to the application of PAI (yellow bar) at $10 \mathrm{pM}$ ( $\mathrm{n}=356 \mathrm{cells}$ from 5 different experiments) and $30 \mathrm{pM}$ ( $\mathrm{n}=293$ cells from 6 different experiments), and recovery after $365 \mathrm{~nm}$ illumination (purple bar). Error bars are \pm SEM. 
PAI (1) and NAI (2) were prepared via two subsequent Menshutkin reactions between the azobenzene linker (4) and the corresponding allo- and orthosteric intermediates (5 and 6, 9) (Scheme 1). Compound $\mathbf{3}$ was synthesized via the typical Mills reaction and successively brominated photochemically to afford the desired linker 4. Notably, this photochemical reaction exempted us from using a radical initiator ${ }^{34}$ and gave an excellent yield (96\%), proving for the first time that light-induced benzylic halogenations can be conveniently used also for the preparation of such versatile photoswitchable linkers. Compounds 5, $\mathbf{6}$ and $\mathbf{9}$ were prepared as previously reported from commercially available starting materials (Scheme 1 and SI). ${ }^{24-26,31}$

As a prerequisite for a reversible light-dependent control of their biological activity, PAI and NAI need to effectively behave as reversible photoswitches, which means that the photoisomerization should be relatively fast and quantitatively significant in both directions. UV/Vis spectroscopy experiments showed that PAI and NAI have the typical absorption bands of conventional azobenzenes. PAl can be isomerized to the cis form (about $73 \%$ conversion) by applying $365 \mathrm{~nm}$ light, while it thermally relaxes back to the trans form in several hours at room temperature. It can be also effectively back-isomerized to the trans form by applying white or blue ( $460 \mathrm{~nm}$ ) light ( $83 \%$ trans) (Fig. 2 and SI, Fig. S2). Surprisingly, NAl resulted refractory to photoisomerization (only $23 \%$ cis after $10 \mathrm{~min}$ at $365 \mathrm{~nm}, \mathrm{SI}$, Fig. S2.1CD), which shows that rational design of azobenzene-containing ligands does not always afford the expected results. We hypothesized that the absorption and emission properties of the naphthalimide moiety ${ }^{35}$ could interfere with its photochromism. Given the unsatisfactorily photochromic behaviour of NAl, we selected only PAI for further studies.

PAl allows reversible photo-activation of $\mathrm{M} 2 \mathrm{mAChRs}$ in calcium imaging experiments and molecular docking simulations. The photopharmacological properties of PAI were first assessed in vitro with real-time calcium imaging assays in transiently transfected HEK cells under 1P-illumination (Fig. 3 and $S I$ ). We tested also the non-photoresponsive muscarinic agonist Iperoxo (IPX) ${ }^{36}$ as a control (Fig. 3a). The application of trans-PAI (dark-adapted state) induced cytosolic calcium oscillations indicative of $M 2$ agonism, which were reduced by converting PAl to its cis form upon illumination with UV light (365 nm) (Fig. 3bc). Calcium oscillations could be restored after back-isomerizing PAl to the trans configuration using blue light $(460 \mathrm{~nm})$. The time course of calcium responses during activation with trans-PAl displayed a diversity of behaviours in individual cells (Fig. 3c), including oscillatory waves, transient peaks, and step responses as previously observed with PLC-activating GPCRs. ${ }^{12}$ Quantification of photoresponses $\left(\Delta F / F_{0}\right)$ to PAI application and $365 \mathrm{~nm}$ illumination shows a reduction in the calcium signal induced by UV light pulses (Fig. 3d). Intriguingly, PAl activated M2 mAChRs in the range of picomolar concentrations, similarly to the super-agonist Iperoxo. ${ }^{36}$ Thus, we demonstrated that PAI can effectively activate M2 mAChRs in vitro in its dark-adapted (trans) form and its activity can be reversibly switched off and on with light.In order to account for the observed photoswitchable activity of PAl in $\mathrm{M} 2 \mathrm{mAChR}$, we looked for putative differences on the receptor level regarding binding efficacy of cis- and trans-PAI using molecular docking simulations (see $S I$ for details). PAI isomers were docked into their theoretical binding site at the human M2 mAChR (PDB 4MQT). Our results suggested that trans-PAl can bind to the $M 2 \mathrm{mAChR}$ in a typical dualsteric pose compatible with receptor activation (SI, Fig. S5.1a). ${ }^{37}$ In contrast, a flipped orientation is favoured in the case of the cis-isomer (SI, Fig. S5.1b). This binding pose is likely incompatible with receptor activation and provides a possible explanation for the light-dependent efficacy of PAI.

Trans-PAl is more effective than cis-PAl at inducing bradycardia and PR lengthening in rats. Once established that PAI allows lightdependent reversible activation of $\mathrm{M} 2 \mathrm{mAChRs}$, we aimed at testing it as an agent to photocontrol cardiac function in vivo. We initially used Wistar rats for our experiments. The intraperitoneal administration of PAI induced progressive bradycardia and PR lengthening in a dose-dependent manner in both configurations (Fig. 4 and SI, Fig. S6.1). These effects were accompanied with
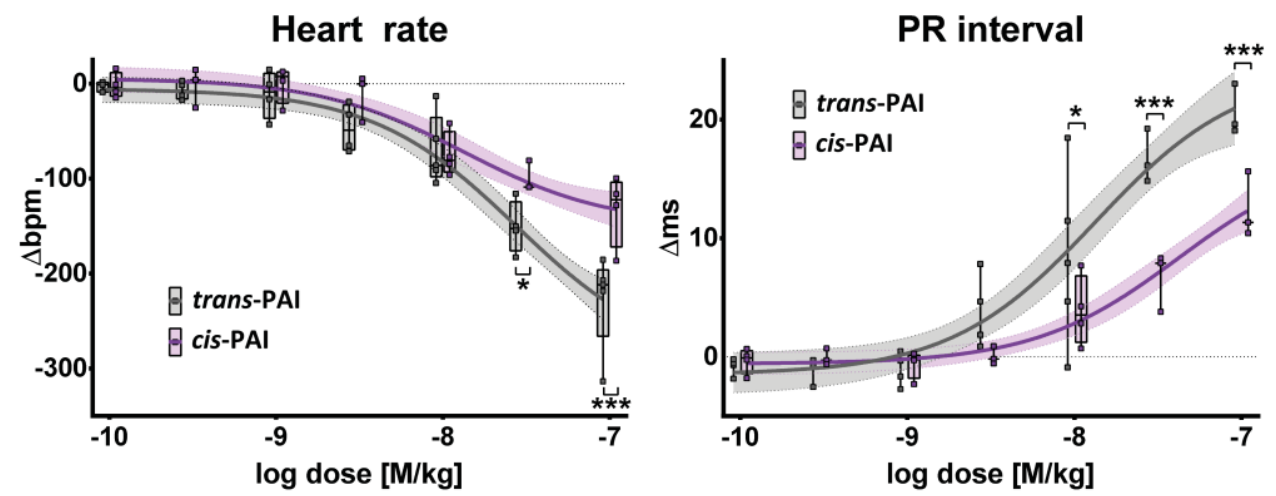

Fig. 4. In vivo effect of trans- and cis-PAI on the cardiac activity of rats. The activity of dark-relaxed (trans, grey plots) and UV-illuminated PAI (cis, purple plots) administered intraperitoneally in anesthetized rats was tested by means of electrocardiography. The heart rate (left panel) and PR interval (right panel) are plotted as a function of increasing doses of both isomers, which induced progressive bradycardia and PR lengthening in a dose-dependent manner. Significant differences between the dark-relaxed and UV-treated PAI were found in the heart rate and PR interval at the higher doses $\left({ }^{*} \mathrm{p}<0.05\right.$; $* * * \mathrm{p}<0.001$ trans vs cis), in agreement with the higher agonist activity of the trans form observed in vitro and in tadpoles. Three rats in the trans-group died because of extreme bradycardia after the $100 \mu \mathrm{M} / \mathrm{kg}$ dose. The effects of PAI were reversible only upon administration of the muscarinic antagonist Atropine. 


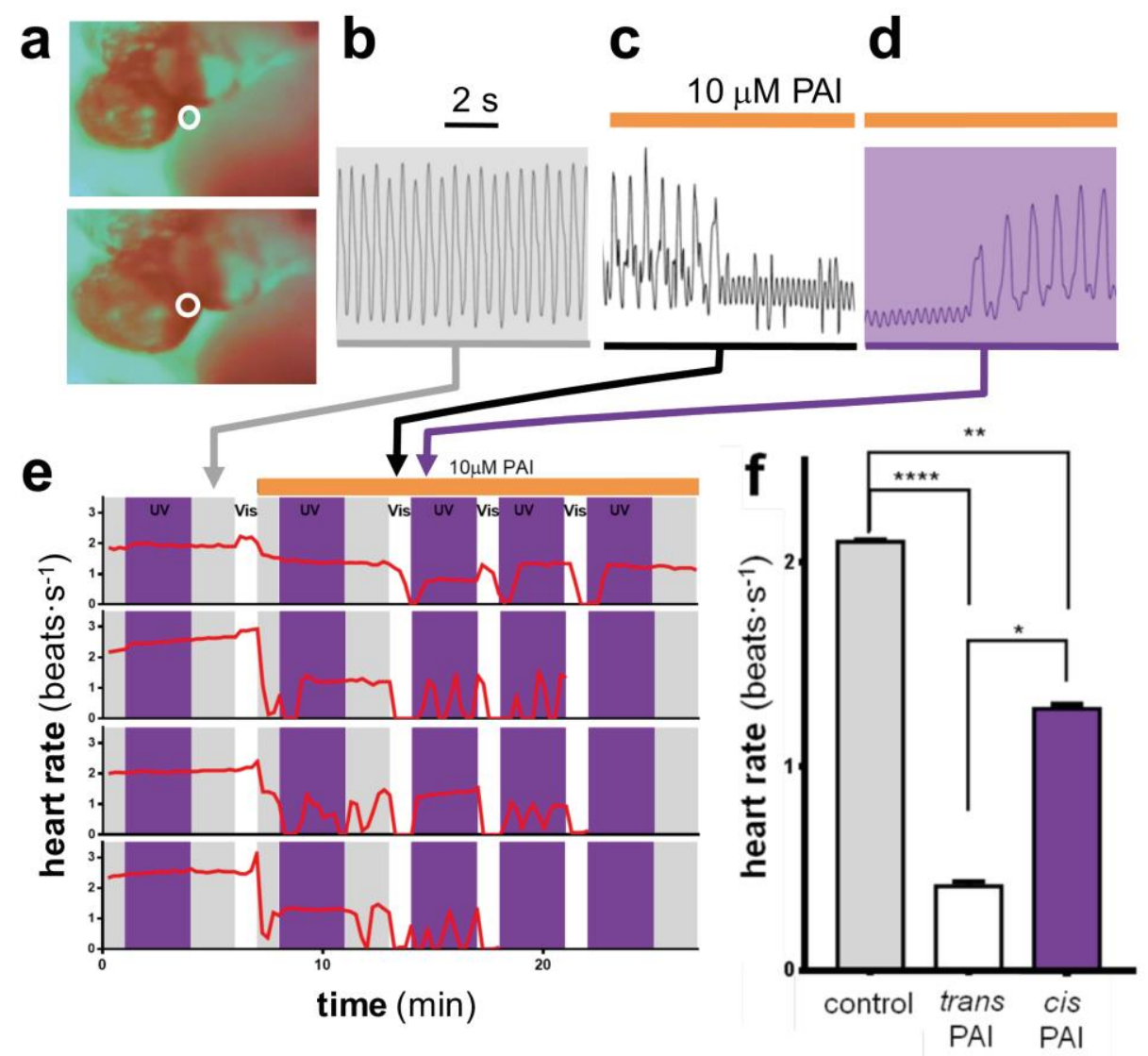

Fig. 5. Photoregulation of heart rate with PAI in frog tadpoles. a) Two video frames of a paralyzed tadpole heart indicating a region of interest (white circle) used to obtain the time course of heart beating movements (average of intensity versus time). b) Normal heartbeat recording obtained in control conditions (dim red light, indicated by a grey shade). Time scale: $2 \mathrm{~s}$. c) Adding $10 \mu \mathrm{M}$ trans-PAI under white light illumination reduces the heartbeat frequency, eventually causing cardiac arrest. d) Under UV illumination (purple shade), trans-PAI is isomerized to cis-PAI and heartbeat is recovered. e) Red traces indicate the heart rate (in beats $\cdot \mathrm{s}-1$, calculated every $15 \mathrm{~s}$ interval) as a function of time in 4 independent experiments with different animals. White, purple, and grey backgrounds indicate illumination with white, UV and dim red light, respectively. Heart rate is not altered by illumination under control conditions (SI Appendix, Fig. S6.2). Adding $10 \mu \mathrm{M}$ trans-PAI under dim light reduces the heart rate in animals 2, 3 and 4 . UV illumination isomerizes PAI to the cis form and the heart rate is partially recovered. Dim red light does not isomerize PAI (SI Appendix, Fig. S6.1) and heart rate is relatively stable. White light converts PAI to the trans isomer, causing cardiac arrest in all 4 animals. UV light restores heartbeat in all animals, some displaying an unstable rate. Several white/UV light cycles were repeated in some animals, showing similar effects. e) Quantification of heart rate during the last minute of each period (beats $\cdot \mathrm{s}-1, \mathrm{n}=4$ tadpoles) in control conditions, under white light (trans-PAI) and under UV light (cis-PAI). f) Two-way for repeated measures ANOVA was performed with uncorrected Fisher's LSD test, significance values were established with a p-value $=0.05$. Error bars represent standard error of the mean (SEM). The heart rate was significantly higher under UV illumination compared to visible light ( -value $<0.05)$. Both isomers produced a significant reduction of heart rate in comparison to controls ( $\mathrm{p}$-value $<0.001$ ).

variable degrees of systemic parasympathetic effects, such as salivation, urination and defecation. At low doses $(\leq 3 \mu \mathrm{M} / \mathrm{kg})$, both isomers yielded a similar small effect, but remarkably differed at intermediate and high doses. At $10 \mu \mathrm{M} / \mathrm{kg}$ PAl and higher doses, the PR interval was significantly more prolonged in trans; at $30 \mu \mathrm{M}$ and higher doses, heart rate was also lower in trans. The effects of PAI could not be photoswitched either with blue or with UV light, showing that the ability of light to penetrate murine cardiac tissue at those wavelengths is likely not sufficient to reach $\mathrm{M} 2 \mathrm{mAChR}$ location. Only the administration of atropine $(2 \mathrm{mg}$ ) completely reverted bradycardia, PR lengthening and systemic parasympathetic effects in both groups (SI, Fig. S6.1). These results demonstrated an enhanced parasympathetic activity for the trans-isomer, and confirmed in mammals the previous findings observed in cells.

PAl enables reversible photocontrol of cardiac activity in Xenopus tropicalis tadpoles. As an alternative to demonstrate reversible control of cardiac function in vivo, we turned to an animal model in which light scattering is known to be low thus allowing better light penetration. We selected Xenopus tropicalis tadpoles for this purpose since they are translucent and are recognized as an excellent model for studying the human cardiovascular system (Fig. 5). ${ }^{38,39}$ Moreover, we had already successfully used video light microscopy to acquire real-time images of the developing beating heart by digitizing the expanding and contracting blood pool in early translucent hearts (Fig. 5a). ${ }^{38,40}$

In the absence of PAl, the cardiac rate of tadpoles remained nearly constant at $2.3 \pm 0.1$ beats $\cdot \mathrm{s}^{-1}$ during control illumination with UV light and at $2.10 \pm 0.01$ beats $\cdot \mathrm{s}^{-1}$ in the dark. The variability score (V.S.) was $7.97 \pm 0.07$. Upon administration of $10 \mu \mathrm{M}$ trans-PAl, heart rate decreased dramatically $\left(0.41 \pm 0.02\right.$ beats $\cdot \mathrm{s}^{-1}$ in the trace of Fig. 5e) leading in some cases to cardiac arrest (Fig. $\mathbf{5 c}$ ). Heart beating recovered progressively upon UV illumination (cisisomerisation, $1.28 \pm 0.02$ beats $\cdot \mathrm{s}^{-1}$, Fig. $\mathbf{5 f}$ ), and was not altered in the dark since thermal relaxation is slow. Some animals displayed less stable cardiac rate during UV periods compared to controls 

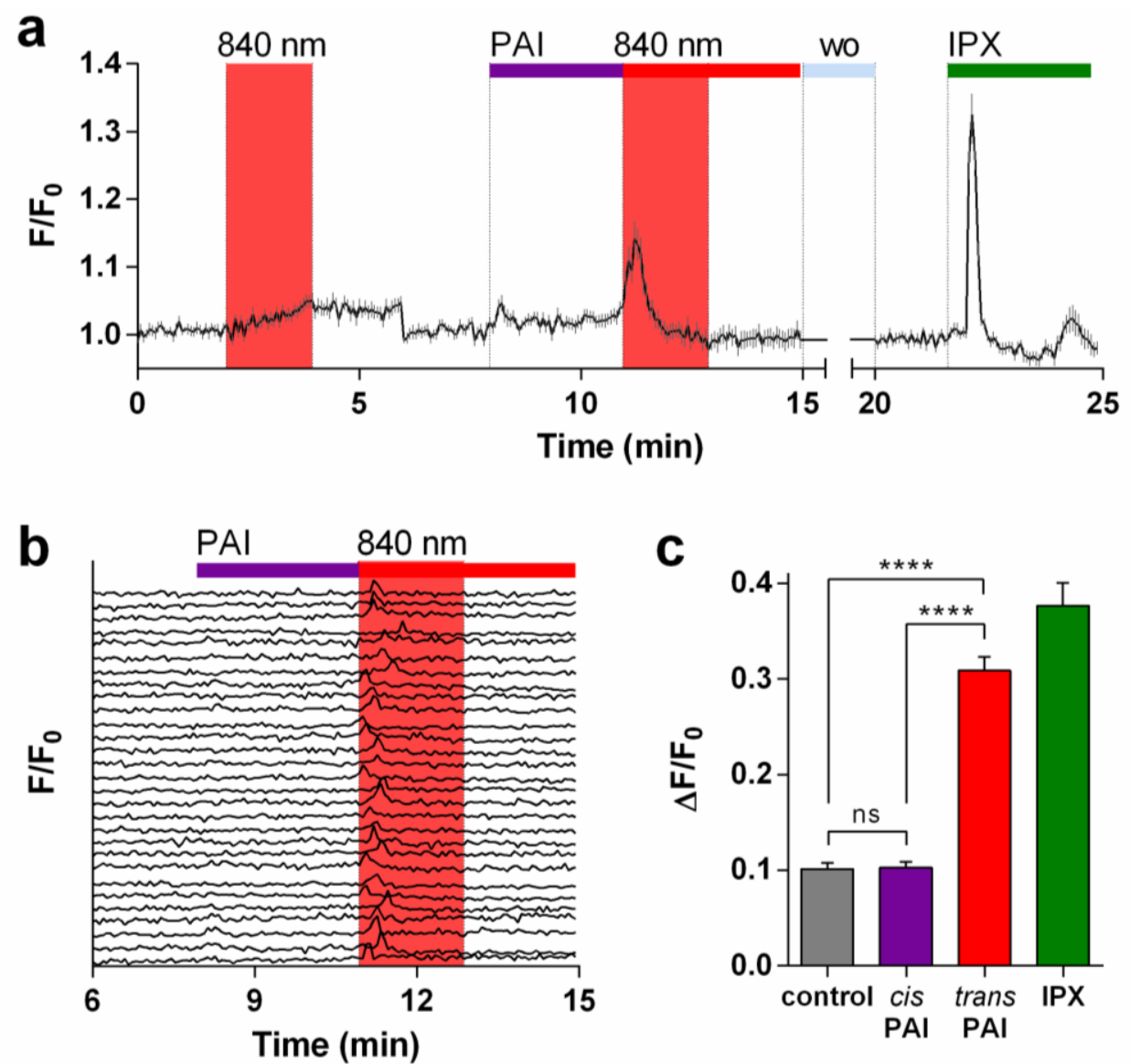

Fig. 6. Activation of PAI with NIR light under $2 \mathrm{P}$ excitation. Real-time calcium imaging traces from HEK cells co-expressing M2 mAChR and GqTOP loaded with $10 \mu \mathrm{M}$ of the calcium indicator OGB1AM. a) Average trace of cell responses to $30 \mathrm{pM}$ cis-PAI pre-irradiated at $365 \mathrm{~nm}$ (purple bar), transenriched PAI obtained under 2P excitation at $840 \mathrm{~nm}$ (red bar), and the muscarinic agonist IPX (green bar) after wash-out (wo, light blue bar) (n=29 cells). Neither 2P excitation alone (red panel) nor cis-PAI elicited calcium responses. Cells gave a sharp response upon application of pulsed NIR light (840 nm) in the presence of PAI as a consequence of its photoisomerization to the trans- active form. IPX was applied as a positive control. Gray shadow in the recordings represents \pm SEM. b) Single cell calcium responses induced by PAI under 2P-excitation at $840 \mathrm{~nm}$ (red panel) (29 cells). Purple bar indicates application of pre-irradiated PAI $(365 \mathrm{~nm})$, red bar indicates illumination at $840 \mathrm{~nm}$. c) Quantification of photoresponses of 29 cells to the application of cis-PAI (purple bar) at $30 \mathrm{pM}$, after switching to trans-PAI using 2P-excitation at $840 \mathrm{~nm}$ (red bar), control under 2P-excitation at $840 \mathrm{~nm}$ (grey bar) and IPX (green bar). Error bars are \pm SEM. Data were analyzed by using one-way ANOVA with Sidak post hoc test for multiple comparisons for statistical significance ( $p$-value $(* * * *)<0.0001$; GraphPad Prism 6).

(V.S. of $17.4 \pm 0.2$ and $8.30 \pm 0.02$, respectively; SI, Fig. S7.3). Subsequent illumination of the animals with visible light (cis-to-trans isomerization) again reduced cardiac rate and eventually interrupted heart beating (V.S. of $374 \pm 47.7$, SI, Fig. S7.3). Cardiac activity was restored by later exposition to UV light, and further UV/visible light cycles confirmed the reversibility of the pharmacological effects. (see example Supporting Movie S1). Overall, these experiments demonstrated that PAI allows remote and reversible control of heart rate with light in living animals.

Activation of PAI with near infrared (NIR) light. In order to overcome the scattering and low penetration of violet and visible illumination, we tested whether PAl could be used to activate M2 mAChRs at longer wavelengths. In fact, a critical aspect that must be addressed to unleash the full potential of light-regulated drugs and favour their translation into clinic is their responsiveness to red or NIR radiation, ${ }^{44,41}$ which enables higher penetration through tissue, abolishes photodamage and, in the case of $2 \mathrm{P}$ excitation, allows three-dimensional subcellular resolution. ${ }^{42-46} \mathrm{PAl}$ has an excellent thermal stability in both configurations (Fig. S2.3) and is photochemically suited for cis-to-trans photoisomerization with NIR light under 2P-excitation, which encouraged us to test its effects in living cells in real-time calcium imaging assays using a confocal microscope equipped with a pulsed laser. (Fig. 6). PAI was initially applied in its cis (off) state, which as expected did not produce cytosolic calcium oscillations. Subsequent illumination at 840 $\mathrm{nm}$ induced robust calcium responses, as previously observed in calcium imaging experiments for cis-to-trans photoisomerization under 1P-excitation (Fig. 6a). These results are quantified in Fig. $6 c$. The responses $(\Delta \mathrm{F} / \mathrm{F} 0)$ obtained for cis-PAI (1P pre-irradiation at $365 \mathrm{~nm}$ ) are comparable to controls, and $2 \mathrm{P}$-induced isomerization to trans-PAl achieves calcium responses nearly as high as perfusion of iperoxo. It is worth noting that even under NIR excitation PAI maintains an outstanding potency (picomolar) to activate M2 mAChRs, which is rarely observed in photoswitches. ${ }^{11,44,47,48}$ Interestingly, 2P microscopy is extensively used for intravital imaging including cardiovascular imaging at subcellular resolution. ${ }^{49,50}$ Thus, PAI has a bright future to control cardiac function with light.

\section{Conclusion}

The rapid and reversible control of cardiac activity is of particular interest in medicine, including the spatiotemporal manipulation of 
close anatomic structures bearing different electrophysiological functions in the heart. Light-activated cardiac drugs could be selectively enhanced in certain regions of the heart (e.g., preventing undesired pro-arrhythmogenic ventricular effects when atria are targeted), or at certain times (on-demand, i.e., active only during atrial fibrillation or bradycardia). For that purpose, cardiac patches with integrated electronics and electric stimulation ${ }^{46}$ could be further equipped with optoelectronic devices for photostimulation. Drug-based cardiac photoregulation techniques offer potential advantages compared to electric stimulation of cardiac muscle, which produces inhomogeneous areas of de- and hyperpolarization, causes faradaic reactions that alter $\mathrm{pH}$, and produce toxic gases $\left(\mathrm{H}_{2}, \mathrm{O}_{2}, \mathrm{Cl}_{2}\right)$, all of which would be prevented by light-stimulation.

To this end, we have developed the first photoswitchable compound that enables control of cardiac activity with light in wildtype animals without genetic manipulation. To the best of our knowledge, PAl is also the first photoswitchable M2 mAChR agonist to be reported. Despite the changes introduced in the ligand structure in order to photoregulate its activity, PAI retains the high potency of its parent compounds Iperoxo and P-8-Iper. ${ }^{31,36}$ PAl activates $M 2$ receptors in its trans configuration and can be reversibly photoswitched with different wavelengths including NIR light under 2P excitation. Future experiments will be addressed to demonstrate that PAI enables precise spatiotemporal control of cardiac function in mammalians in combination with $2 \mathrm{P}$ cardiovascular imaging.

\section{ASSOCIATED CONTENTS}

\section{Supporting Information}

The Supporting Information is available free of charge on the ACS Publications website. Detailed materials and methods, synthetic procedures, chemical analyses, and any additional data and figures as noted in the text (PDF).

\section{Conflicts of interest}

There are no conflicts to declare.

\section{Authors information}

Corresponding Author

* Prof. Pau Gorostiza, Institute for Bioengineering of Catalonia (IBEC), Barcelona Institute for Science and Technology (BIST), Carrer de Baldiri Reixac 15-21, 08028 Barcelona, Spain. E-mail: pau@icrea.cat.

\section{Author Contributions}

FR performed PAI synthesis and characterization, in vitro and in vivo experiments. CM designed PAl compound, supervised synthesis and characterization, and performed in silico docking simula-tions. AGC, RS, EC, and RM performed in vitro experiments. AG per-formed in vivo experiments in frog tadpoles. LA performed PAI synthesis and characterization. MB performed in vivo experiments in rats. $\mathrm{MD}$ and $\mathrm{UH}$ supervised $\mathrm{PAI}$ synthesis and characterization. EG designed and performed in vivo experiments in rats. PG conceived the project and designed experiments. $F R, C M$, and PG wrote the paper with contributions from all authors. All authors have given approval to the final version of the manuscript. $\uparrow$ Fabio Riefolo and Carlo Matera contributed equally. All authors have given approval to the final version of the manuscript.

\section{Funding Sources}

This project has received funding from the EU Horizon 2020 Framework Programme for Research and Innovation under the Specific Grant Agreement 2 (Human Brain Project WaveScalES SGA2 No. 785907), AGAUR/Generalitat de Catalunya (CERCA Programme, 2017-SGR-1442 and 2017-SGR-1548), FEDER funds, ERANET SynBio MODULIGHTOR, Fundaluce foundation, Ramón Areces foundation, MINECO (FPI fellowship BES-2014-068169 and project CTQ2016-80066R), and CATCH-ME (grant agreement no 633196). CM was supported by the Ermenegildo Zegna Founder's Scholarship. LA was supported by the graduate program "Receptor Dynamics - Emerging paradigms for novel drugs" funded by the Elite Network of Bavaria.

\section{Notes}

All animal procedures were authorized by the local ethics review boards (IDIBAPS-Hospital Clínic de Barcelona, n.92117, 02/03/2017; Comitè Ètic d'Experimentació Animal de la Universitat de Barcelona, n.306/18, 25/06/2018).

\section{ACKNOWLEDGMENTS.}

The authors are grateful to Jean-Philippe Pin for providing the chimeric $\mathrm{Gi} / \mathrm{Gq}$ protein clone and to Núria Camarero for helping during preliminary in vitro experiments. $\mathrm{CM}$ and FR are grateful to Prof. Marco De Amici for helpful discussion and continuous support. Molecular graphics and analyses were performed with the UCSF Chimera package. Chimera is developed by the Resource for Biocomputing, Visualization, and Informatics at the University of California, San Francisco (supported by NIGMS P41-GM103311). Mass spectrometry was performed at the IRB Barcelona Mass Spectrometry Core Facility, which actively participates in the BMBS European COST Action BM 1403 and is a member of Proteored, PRB2-ISCIII, supported by grant PRB2 (IPT13/0001 - ISCIIISGEFI / FEDER).

\section{REFERENCES}

(1) Fabritz, L.; Guasch, E.; Antoniades, C.; Bardinet, I.; Benninger, G.; Betts, T. R.; Brand, E.; Breithardt, G.; Bucklar-Suchankova, G.; Camm, A. J.; et al. Expert Consensus Document: Defining the Major Health Modifiers Causing Atrial Fibrillation: A Roadmap to Underpin Personalized Prevention and Treatment. Nat. Rev. Cardiol. 2016, 13 (4), 230-237.

Yun, S. H.; Kwok, S. J. J. Light in Diagnosis, Therapy and Surgery. Nat. Biomed. Eng. 2017, 1 (1), 8.

Pianca, N.; Zaglia, T.; Mongillo, M. Will Cardiac Optogenetics Find the Way through the Obscure Angles of Heart Physiology? Biochem. Biophys. Res. Commun. 2017, 482 (4), 515-523.

(4) Yu, L.; Zhou, L.; Cao, G.; Po, S. S.; Huang, B.; Zhou, X.; Wang, M.; Yuan, S.; Wang, Z.; Wang, S.; et al. Optogenetic Modulation of Cardiac Sympathetic Nerve Activity to Prevent Ventricular Arrhythmias. J. Am. Coll. Cardiol. 2017, 70 (22), 2778-2790.

(5) Bingen, B. O.; Engels, M. C.; Schalij, M. J.; Jangsangthong, W.; Neshati, Z.; Feola, I.; Ypey, D. L.; Askar, S. F. A.; Panfilov, A. V.; Pijnappels, D. A.; et al. Light-Induced Termination of Spiral Wave Arrhythmias by Optogenetic Engineering of Atrial Cardiomyocytes. Cardiovasc. Res. 2014, 104 (1), 194-205.

(6) Bruegmann, T.; Boyle, P. M.; Vogt, C. C.; Karathanos, T. V; Arevalo, H. J.; Fleischmann, B. K.; Trayanova, N. A.; Sasse, P. Optogenetic Defibrillation Terminates Ventricular Arrhythmia in 
Mouse Hearts and Human Simulations. J. Clin. Invest. 2016, 126 (10), 3894-3904.

(7) Boyle, P. M.; Karathanos, T. V; Trayanova, N. A. "Beauty Is a Light in the Heart": The Transformative Potential of Optogenetics for Clinical Applications in Cardiovascular Medicine. Trends Cardiovasc. Med. 2015, 25 (2), 73-81.

(8) Lerch, M. M.; Hansen, M. J.; van Dam, G. M.; Szymanski, W.; Feringa, B. L. Emerging Targets in Photopharmacology. Angew. Chem. Int. Ed. Engl. 2016, 55 (37), 10978-10999.

(9) Izquierdo-Serra, M.; Trauner, D.; Llobet, A.; Gorostiza, P. Optical Modulation of Neurotransmission Using Calcium Photocurrents through the Ion Channel LiGluR. Front. Mol. Neurosci. 2013, 6, 3.

(10) Nevola, L.; Martín-Quirós, A.; Eckelt, K.; Camarero, N.; Tosi, S.; Llobet, A.; Giralt, E.; Gorostiza, P. Light-Regulated Stapled Peptides to Inhibit Protein-Protein Interactions Involved in Clathrin-Mediated Endocytosis. Angew. Chemie Int. Ed. 2013, 52 (30), 7704-7708.

(11) Izquierdo-Serra, M.; Gascón-Moya, M.; Hirtz, J. J.; Pittolo, S.; Poskanzer, K. E.; Ferrer, È.; Alibés, R.; Busqué, F.; Yuste, R.; Hernando, J.; et al. Two-Photon Neuronal and Astrocytic Stimulation with Azobenzene-Based Photoswitches. J. Am. Chem. Soc. 2014, 136 (24), 8693-8701.

(12) Pittolo, S.; Gómez-Santacana, X.; Eckelt, K.; Rovira, X.; Dalton, J.; Goudet, C.; Pin, J.-P.; Llobet, A.; Giraldo, J.; Llebaria, A.; et al. An Allosteric Modulator to Control Endogenous $G$ Protein-Coupled Receptors with Light. Nat. Chem. Biol. 2014, 10 (10), 813-815.

(13) Izquierdo-Serra, M.; Bautista-Barrufet, A.; Trapero, A.; GarridoCharles, A.; Díaz-Tahoces, A.; Camarero, N.; Pittolo, S.; Valbuena, S.; Pérez-Jiménez, A.; Gay, M.; et al. Optical Control of Endogenous Receptors and Cellular Excitability Using Targeted Covalent Photoswitches. Nat. Commun. 2016, 7, 12221.

(14) Velema, W. A.; Szymanski, W.; Feringa, B. L. Photopharmacology: Beyond Proof of Principle. J. Am. Chem. Soc. 2014, 136 (6), 2178-2191.

(15) Matera, C.; Gomila, A. M. J.; Camarero, N.; Libergoli, M.; Soler, C.; Gorostiza, P. Photoswitchable Antimetabolite for Targeted Photoactivated Chemotherapy. J. Am. Chem. Soc. 2018, 140 (46), 15764-15773.

(16) Hamaoka, T.; McCully, K. K.; Quaresima, V.; Yamamoto, K.; Chance, B. Near-Infrared Spectroscopy/imaging for Monitoring Muscle Oxygenation and Oxidative Metabolism in Healthy and Diseased Humans. J. Biomed. Opt. 2007, 12 (6), 62105.

(17) Taub, A. F. Photodynamic Therapy in Dermatology: History and Horizons. J. Drugs Dermatol. 3 (1 Suppl), S8-25.

(18) Kale, R. P.; Kouzani, A. Z.; Walder, K.; Berk, M.; Tye, S. J. Evolution of Optogenetic Microdevices. Neurophotonics 2015, 2 (3), 31206.

(19) Brodde, O. E.; Michel, M. C. Adrenergic and Muscarinic Receptors in the Human Heart. Pharmacol. Rev. 1999, 51 (4), 651-690.

(20) Brodde, O.-E.; Bruck, H.; Leineweber, K.; Seyfarth, T. Presence, Distribution and Physiological Function of Adrenergic and Muscarinic Receptor Subtypes in the Human Heart. Basic Res. Cardiol. 2001, 96 (6), 528-538.

(21) Dhein, S.; van Koppen, C. J.; Brodde, O. E. Muscarinic Receptors in the Mammalian Heart. Pharmacol. Res. 2001, 44 (3), 161-182.

(22) Caulfield, M. P.; Birdsall, N. J. International Union of Pharmacology. XVII. Classification of Muscarinic Acetylcholine Receptors. Pharmacol. Rev. 1998, 50 (2), 279-290.

(23) De Amici, M.; Dallanoce, C.; Holzgrabe, U.; Tränkle, C.; Mohr, K. Allosteric Ligands for G Protein-Coupled Receptors: A Novel Strategy with Attractive Therapeutic Opportunities. Med. Res. Rev. 2009, 30 (3), 463-549.

(24) Mohr, K.; Tränkle, C.; Kostenis, E.; Barocelli, E.; De Amici, M.; Holzgrabe, U. Rational Design of Dualsteric GPCR Ligands: Quests and Promise. Br. J. Pharmacol. 2010, 159 (5), 997-1008.

(25) Disingrini, T.; Muth, M.; Dallanoce, C.; Barocelli, E.; Bertoni, S.; Kellershohn, K.; Mohr, K.; De Amici, M.; Holzgrabe, U. Design, Synthesis, and Action of Oxotremorine-Related Hybrid-Type Allosteric Modulators of Muscarinic Acetylcholine Receptors. J. Med. Chem. 2006, 49 (1), 366-372.
Antony, J.; Kellershohn, K.; Mohr-Andrä, M.; Kebig, A.; Prilla, S.; Muth, M.; Heller, E.; Disingrini, T.; Dallanoce, C.; Bertoni, S.; et al. Dualsteric GPCR Targeting: A Novel Route to Binding and Signaling Pathway Selectivity. FASEB J. 2009, 23 (2), 442-450.

(27) Matera, C.; Tata, A. M. Pharmacological Approaches to Targeting Muscarinic Acetylcholine Receptors. Recent Pat. CNS Drug Discov. 2014, 9 (2), 85-100.

(28) Agnetta, L.; Kauk, M.; Canizal, M. C. A.; Messerer, R.; Holzgrabe, U.; Hoffmann, C.; Decker, M. A Photoswitchable Dualsteric Ligand Controlling Receptor Efficacy. Angew. Chem. Int. Ed. Engl. 2017, 56 (25), 7282-7287.

(29) De Min, A.; Matera, C.; Bock, A.; Holze, J.; Kloeckner, J.; Muth, M.; Traenkle, C.; De Amici, M.; Kenakin, T.; Holzgrabe, U.; et al. A New Molecular Mechanism To Engineer Protean Agonism at a G Protein-Coupled Receptor. Mol. Pharmacol. 2017, 91 (4), 348356.

(30) Bock, A.; Merten, N.; Schrage, R.; Dallanoce, C.; Bätz, J.; Klöckner, J.; Schmitz, J.; Matera, C.; Simon, K.; Kebig, A.; et al. The Allosteric Vestibule of a Seven Transmembrane Helical Receptor Controls G-Protein Coupling. Nat. Commun. 2012, 3, 1044.

(31) Matera, C.; Flammini, L.; Quadri, M.; Vivo, V.; Ballabeni, V.; Holzgrabe, U.; Mohr, K.; De Amici, M.; Barocelli, E.; Bertoni, S.; et al. Bis(ammonio)alkane-Type Agonists of Muscarinic Acetylcholine Receptors: Synthesis, in Vitro Functional Characterization, and in Vivo Evaluation of Their Analgesic Activity. Eur. J. Med. Chem. 2014, 75, 222-232.

(32) Cristofaro, I.; Spinello, Z.; Matera, C.; Fiore, M.; Conti, L.; De Amici, M.; Dallanoce, C.; Tata, A. M. Activation of M2 Muscarinic Acetylcholine Receptors by a Hybrid Agonist Enhances Cytotoxic Effects in GB7 Glioblastoma Cancer Stem Cells. Neurochem. Int. 2018, 118, 52-60.

(33) Broichhagen, J.; Frank, J. A.; Trauner, D. A Roadmap to Success in Photopharmacology. Acc. Chem. Res. 2015, 48 (7), 1947-1960.

(34) Tolosa, J.; Kub, C.; Bunz, U. H. F. Hyperbranched: A Universal Conjugated Polymer Platform. Angew. Chemie Int. Ed. 2009, 48 (25), 4610-4612.

(35) Jacquemin, D.; Perpète, E. A.; Scalmani, G.; Ciofini, I.; Peltier, C.; Adamo, C. Absorption and Emission Spectra of 1,8Naphthalimide Fluorophores: A PCM-TD-DFT Investigation. Chem. Phys. 2010, 372 (1-3), 61-66.

(36) Schrage, R.; Seemann, W. K.; Klöckner, J.; Dallanoce, C.; Racké, K.; Kostenis, E.; De Amici, M.; Holzgrabe, U.; Mohr, K. Agonists with Supraphysiological Efficacy at the Muscarinic M2 ACh Receptor. Br. J. Pharmacol. 2013, 169 (2), 357-370.

(37) Bock, A.; Bermudez, M.; Krebs, F.; Matera, C.; Chirinda, B.; Sydow, D.; Dallanoce, C.; Holzgrabe, U.; De Amici, M.; Lohse, M. J.; et al. Ligand Binding Ensembles Determine Graded Agonist Efficacies at a G Protein-Coupled Receptor. J. Biol. Chem. 2016, 291 (31), 16375-16389.

(38) Bartlett, H. L.; Scholz, T. D.; Lamb, F. S.; Weeks, D. L. Characterization of Embryonic Cardiac Pacemaker and Atrioventricular Conduction Physiology in Xenopus Laevis Using Noninvasive Imaging. Am. J. Physiol. Heart Circ. Physiol. 2004, 286 (6), H2035-41.

(39) Nieuwkoop P., F. J. Normal Table of Xenopus Laevis (Daudin): A Systematical \& Chronological Survey of the Development from the Fertilized Egg till the End of Metamorphosis; 1994.

(40) Eckelt, K.; Masanas, H.; Llobet, A.; Gorostiza, P. Automated HighThroughput Measurement of Body Movements and Cardiac Activity of Xenopus Tropicalis Tadpoles. J. Biol. Methods 2014, 1 (2), 9.

(41) Dong, M.; Babalhavaeji, A.; Samanta, S.; Beharry, A. A.; Woolley, G. A. Red-Shifting Azobenzene Photoswitches for in Vivo Use. Acc. Chem. Res. 2015, 48 (10), 2662-2670.

(42) Pawlicki, M.; Collins, H. A.; Denning, R. G.; Anderson, H. L. TwoPhoton Absorption and the Design of Two-Photon Dyes. Angew. Chem. Int. Ed. Engl. 2009, 48 (18), 3244-3266.

(43) Bort, G.; Gallavardin, T.; Ogden, D.; Dalko, P. I. From One-Photon to Two-Photon Probes: \&quot;caged\&quot; Compounds, Actuators, and Photoswitches. Angew. Chem. Int. Ed. Engl. 2013 52 (17), 4526-4537. 
(44) Cabré, G.; Garrido-Charles, A.; Moreno, M.; Bosch, M.; Porta-dela-Riva, M.; Krieg, M.; Gascón-Moya, M.; Camarero, N.; Gelabert, R.; Lluch, J. M.; et al. Rationally Designed Azobenzene Photoswitches for Efficient Two-Photon Neuronal Excitation. Nat. Commun. 2019, 10 (1), 907.

(45) Pittolo, S.; Lee, H.; Lladó, A.; Tosi, S.; Bosch, M.; Bardia, L.; Gómez-Santacana, X.; Llebaria, A.; Soriano, E.; Colombelli, J.; et al. Reversible Silencing of Endogenous Receptors in Intact Brain Tissue Using Two-Photon Pharmacology. bioRxiv 2019, 515288.

(46) Feiner, R.; Engel, L.; Fleischer, S.; Malki, M.; Gal, I.; Shapira, A.; Shacham-Diamand, Y.; Dvir, T. Engineered Hybrid Cardiac Patches with Multifunctional Electronics for Online Monitoring and Regulation of Tissue Function. Nat. Mater. 2016, 15 (6), 679-685.

Passlick, S.; Richers, M. T.; Ellis-Davies, G. C. R.
Neurons with One- and Two-Photon Excitation. Angew. Chem Int. Ed. Engl. 2018, 57 (38), 12554-12557.

(48) Bartels, E.; Wassermann, N. H.; Erlanger, B. F.; Kienzler, M. A.; Yuan, Z.; Madsen, D.; Larsen, D. S.; Isacoff, E. Y. Photochromic Activators of the Acetylcholine Receptor. Proc. Natl. Acad. Sci. U. S. A. 1971, 68 (8), 1820-1823.

(49) Scherschel, J. A.; Rubart, M. Cardiovascular Imaging Using TwoPhoton Microscopy. Microsc. Microanal. 2008, 14 (6), 492-506.

(50) Matsuura, R.; Miyagawa, S.; Fukushima, S.; Goto, T.; Harada, A.; Shimozaki, Y.; Yamaki, K.; Sanami, S.; Kikuta, J.; Ishii, M.; et al. Intravital Imaging with Two-Photon Microscopy Reveals Cellular Dynamics in the Ischeamia-Reperfused Rat Heart. Sci. Rep. 2018, $8(1), 15991$. Thermodynamically Stable, Photoreversible Pharmacology in 


\section{Graphical Abstract}

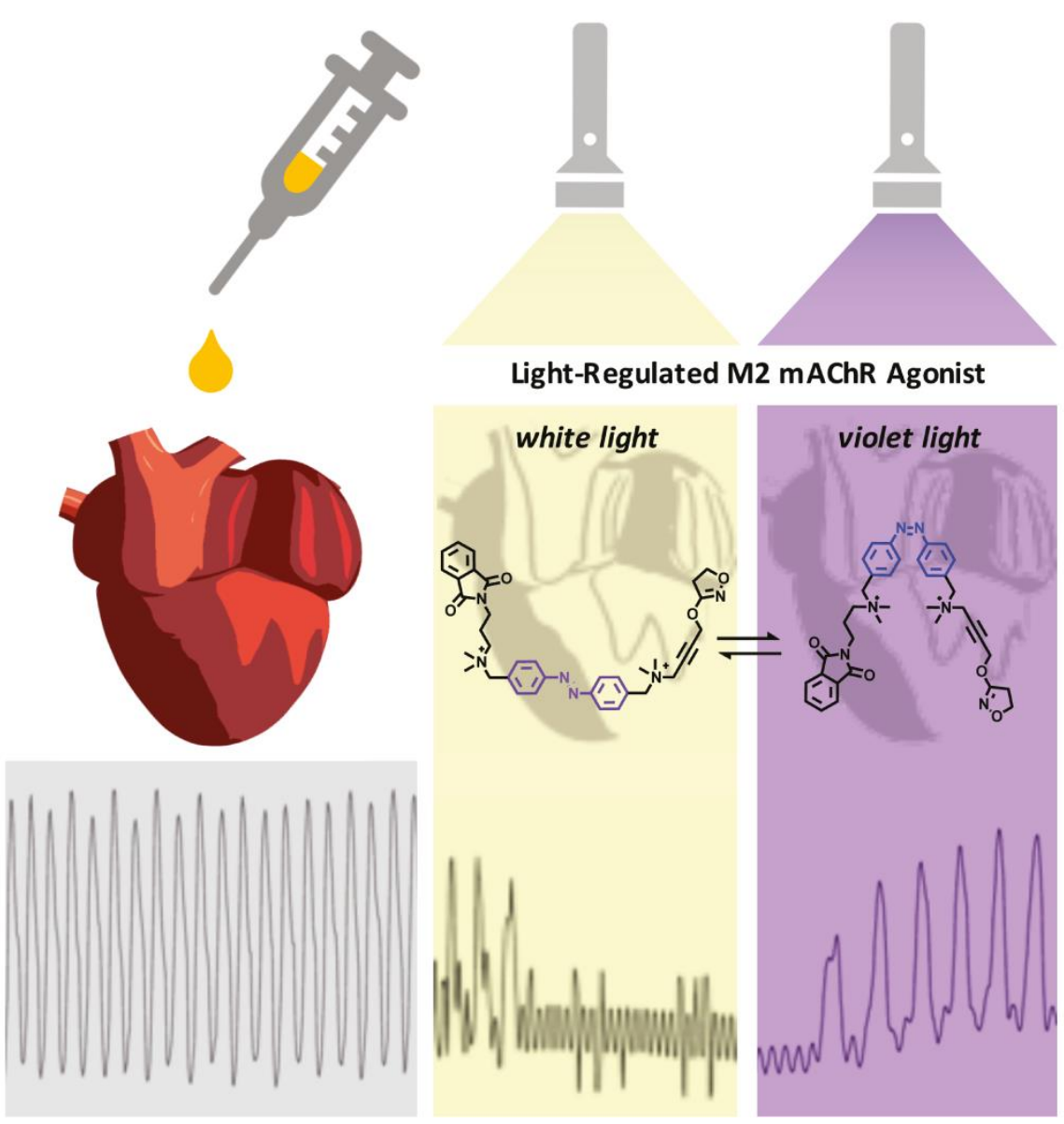

